\title{
The Effect of Preincubation of Seed Crystals of Uric Acid and Monosodium Urate with Undiluted Human Urine to Induce Precipitation of Calcium Oxalate in Vitro: Implications for Urinary Stone Formation
}

\author{
Phulwinder K. Grover and Rosemary L. Ryall \\ Division of Urology, Department of Surgery, Flinders University School of Medicine, Flinders Medical \\ Centre, Bedford Park, South Australia, AU \\ Accepted July 22, 2002
}

\begin{abstract}
Background: Previous studies demonstrated that crystals of uric acid (UA) and sodium urate (NaU) can induce the precipitation of calcium oxalate $(\mathrm{CaOx})$ from its inorganic metastable solutions, but similar effects have not been unequivocally shown to occur in urine. The aim of this investigation was to determine whether preincubation of these seeds with urine alter their ability to induce deposition of $\mathrm{CaOx}$ from solution and thus provide a possible explanation for discrepancy of results obtained from aqueous inorganic solutions and undiluted urine.

Materials and Methods: The effects of commercial seed crystals of UA, NaU and $\mathrm{CaOx}(6 \mathrm{mg} / 100 \mathrm{ml})$ on CaOx crystallization were compared in a solution with the same crystals that had been preincubated for 3 hours with healthy male urine. A Coulter Counter was used to follow the crystallization and decrease in soluble ${ }^{14} \mathrm{C}$-oxalate was measured to determine the deposition of CaOx. The precipitated particles were examined by scanning electron microscopy (SEM). The preincubated seeds were demineralized and proteins released were analyzed by sodium dodecylsulphate polyacrylamide gel electrophoresis (SDS-PAGE).

Results: Analysis of ${ }^{14} \mathrm{C}$-oxalate data revealed that while treated UA seeds did not affect $\mathrm{CaOx}$ deposition, those of $\mathrm{NaU}$ and $\mathrm{CaOx}$ inhibited the process by $51.9(p \leq 0.05)$ and $8.5 \%(p \leq 0.05)$ relative to their respective untreated
\end{abstract}

counterparts. Particle size analysis showed that the average modal sizes of particles precipitated in the presence of treated seed crystals of UA, NaU, and CaOx were very similar to those deposited in the presence of their respective untreated controls. These findings were confirmed by SEM which also showed that seed crystals of UA and NaU, untreated and treated, were attached like barnacles upon the surfaces of $\mathrm{CaOx}$ crystals which themselves were bigger than those precipitated in the presence of $\mathrm{CaOx}$ seeds. SDS-PAGE analysis of the demineralized treated seeds showed that they all selectively adsorbed urinary proteins, and perhaps other urinary macromolecules and low molecular weight components, on their surface.

Conclusions: It was concluded that preincubation with urine, such as occurs in vivo, only slightly reduces the ability of seed crystals of CaOx, but not of UA, to cause deposition of $\mathrm{CaOx}$. The most striking effect was on $\mathrm{NaU}$ seeds where the preincubation quite dramatically attenuated their promotory effect on the mineral deposition. This may explain the discrepancy between findings of studies carried out in inorganic solutions and undiluted human urine. This stresses the invalidity of directly extrapolating results obtained in inorganic solutions to likely effects in urine and more importantly, on stone formation.

\section{Introduction}

Hyperuricosuria has long been documented as a predisposing factor to calcium oxalate $(\mathrm{CaOx})$ stone formation. This association is so strong that some clinicians even regard "hyperuricosuric calcium oxalate" stone disease as a separate clinical entity. A critical review of the literature revealed that this is based primarily on empirical clinical evidence (reviewed in 1-3), the most significant of which is that administration of allopurinol, a drug which reduces urinary out put of urate, reduces the recurrence of

Address correspondence and reprint requests to: P. K. Grover, Division of Urology, Department of Surgery, Flinders Medical Centre, Bedford Park, South Australia 5042, Australia, Phone: 61 8-8204-4870, fax: 61 8-8374-0832,

e-mail: pk.Grover@flinders.edu.au
CaOx stone formation in patients whose only demonstrable abnormality is hyperuricosuria (4-11). In an attempt to explain this causal relationship of hyperuricosuria to $\mathrm{CaOx}$ stone formation, one mechanism that has been most commonly cited is epitaxy. The term epitaxy was first coined by Royer as the growth of one crystal type upon the crystalline surface of another (12). The process was first invoked by Modlin (13) as a possible mechanism for the formation of urinary stones that usually consist of a mixture of different minerals. One year later, Lonsdale (14) demonstrated, using X-ray crystallography, the existence of several crystal lattice fits for anhydrous uric acid, uric acid dihydrate, $\mathrm{CaOx}$ monohydrate (whewellite), and $\mathrm{CaOx}$ dihydrate (weddelite). She theorized epitaxy as a mechanism of stone formation and to explain the encapsulation 
of a crystal core by alternating layers of other minerals-a pattern commonly seen in urinary calculi. The theory awaited experimental verification till 1975 when Coe et al. (15) and Pak and Arnold (16) reported that urate seed crystals enhanced the precipitation of $\mathrm{CaOx}$ from an inorganic metastable solution of this salt. These observations were later confirmed by independent groups (17-26). Collectively, the findings once again focused attention on epitaxy as a possible mechanism to link hyperuricosuria to $\mathrm{CaOx}$ stone formation, and, more importantly, a justification for administering allopurinol to reduce recurrence of $\mathrm{CaOx}$ stone formation. However, although the data proved conclusively that epitaxial deposition of $\mathrm{CaOx}$ upon urate seed crystals can occur under inorganic reaction conditions, they are largely academic from a pathophysiological perspective, because stones are formed in urine which, in addition to common inorganic constituents, also contains a wide selection of organic macromolecules whose possible effects on the process have never been studied. Furthermore, the results of our previous study revealed that the promotory effect of uric acid and monosodium urate seeds does not occur to physiologically significant degree in undiluted human urine (27). This strongly militates against credibility of the epitaxy theory.

It is now well documented that urinary macromolecules bind crystals in a selective manner and thus participate in the crystallization process (28). In an attempt to clarify the possible role of epitaxy in stone formation, the aim of the present investigation was to determine whether preincubation of seed crystals of uric acid and monosodium urate with urine alter their ability to induce deposition of $\mathrm{CaOx}$ from its aqueous inorganic solution, and thereby provide a possible explanation for the disparity of results obtained with synthetic inorganic solutions and undiluted human urine. Crystals of $\mathrm{CaOx}$ were also included as a basis for comparison.

\section{Materials and Methods Materials}

Uric acid (UA), monosodium urate (NaU), calcium chloride dihydrate $\left(\mathrm{CaCl}_{2} 2 \mathrm{H}_{2} \mathrm{O}\right)$, ethylenediaminetetraacetic acid (EDTA), 2-mercaptoethanol, morpholinoethanesulfonic acid (MES) and tris(hydroxymethyl) aminomethane (Trizma-base) were purchased from Sigma Chemical Company, St. Louis, MO, USA. Calcium oxalate $(\mathrm{CaOx})$ monohydrate, sodium oxalate and hydrochloric acid $(\mathrm{HCl})$ were from $\mathrm{BDH}$ Chemicals Ltd. Poole, England. Sodium chloride $(\mathrm{NaCl})$ and sodium azide were from E. Merck, Darmstadt, Germany. Bio-Gel P-6 DG was purchased from Bio-Rad Laboratories, Richmond, CA, USA. ${ }^{14} \mathrm{C}$-oxalic acid was from NEN Products, Boston, MA, USA. All other chemicals and materials used in this study were of the highest purity available.
Preparation of Preincubated Seed Crystals of UA, NaU, and $\mathrm{CaOx}$ with Urine

Collection and Preparation of Urine Samples Twenty-four hour urine samples were collected without preservative from five healthy men (mean age 40 years) who had no previous history of urinary stone disease. The samples were refrigerated during the collection period and during storage before use. Absence of blood from the specimens was confirmed using Multistix test strips (Miles Laboratories, Mulgrave, Victoria, Australia) and the samples were pooled. The pooled sample was centrifuged using a JA-14 fixed-angle rotor at $8000 \times g$ for $15 \mathrm{~min}$ at $20^{\circ} \mathrm{C}$ in a Beckman $\mathrm{J} 2-21 \mathrm{M} / \mathrm{E}$ centrifuge (Beckman Instruments, Palo Alto, CA, USA). The supernatant was filtered through $0.22 \mu \mathrm{m}$ Millipore filters (\# GVWP 142 50, Millipore Corporation, Bedford, MA, USA). An aliquot (approximately $50 \mathrm{ml}$ ) of the filtrate was transferred to dialysis bag (with nominal molecular weight cut-off of $10 \mathrm{kDa}$ ) and the remaining filtrate was divided into three equal portions. The dialysis bag was stirred at $4^{\circ} \mathrm{C}$ in cold room in a conical glass flask containing 6 litre deionized water. The dialysis was continued for 36 hours with dialyzate changed every 12 hours after which the sample was applied to a Bio-Gel P-6 DG desalting column. The protein peak was collected, lyophilized and stored at $-20^{\circ} \mathrm{C}$ until further use.

Preincubation of Seed Crystals of UA, NaU, and $\mathrm{CaOX}$ with Urine Commercial preparations of seed crystals of UA, NaU, and CaOx were added, separately, to the remaining three aliquots of the urine sample such that the final seed concentration in each was $1 \mathrm{~g} / \mathrm{l}$. The samples were stirred for 3 hours at room temperature using teflon coated magnetic stir bar in conical glass flasks. The crystals were harvested by filtration through $0.22 \mu \mathrm{m}$ Millipore filters and washed thoroughly with deionized water. They were then lyophilized and stored at $-20^{\circ} \mathrm{C}$ until required. These seed crystals will be referred to as "treated".

The control seed crystals were from the same commercial batches of UA, NaU, and CaOx seeds as used above and were not treated in any way. These seed crystals will be referred to as "untreated."

\section{Crystallization Experiments}

Measurement of Crystallization by Coulter Counter Analysis Initial attempts to study the effect of the seed crystals on $\mathrm{CaOx}$ crystallization were made in the reaction medium as described by Pak and Arnold (16). Briefly, the seed crystals of UA, NaU, and $\mathrm{CaOx}$, untreated and treated, were separately added to the reaction medium to give a final suspension concentration of $6 \mathrm{mg} / 100 \mathrm{ml}$, and the samples were incubated in a shaking water-bath at $37^{\circ} \mathrm{C}$ : seed crystals of $\mathrm{UA}$ and $\mathrm{NaU}$, both untreated and treated, dissolved in 15-30 minutes. Similar dissolution was also observed using the reaction 
medium as described by Coe et al. (15). For this reason alone, the effect of seed crystals in the present investigation was studied in a slightly modified reaction medium employing the same calcium and oxalate concentrations as used by Coe et al. (15). In brief, a solution containing $5 \mathrm{mM} \mathrm{CaCl} 22_{2} \mathrm{H}_{2} \mathrm{O}$, $10 \mathrm{mM}$ morpholinoethanesulfonic acid, $0.15 \mathrm{M} \mathrm{NaCl}$ was adjusted to $\mathrm{pH} 6.00$ and saturated with urate by stirring at $37^{\circ} \mathrm{C}$ first with commercial preparations of UA followed by NaU crystals (to prevent dissolution of these seeds during their subsequent incubation in the reaction medium). The saturated solution was $0.22 \mu \mathrm{m}$ filtered and divided into seven aliquots.

Untreated and treated seed crystals of UA, NaU, and $\mathrm{CaOx}$ were ground in an agate mortar to remove large clumps. Slurries containing $6 \mathrm{mg} / \mathrm{ml}$ of the seeds were prepared in $0.15 \mathrm{M} \mathrm{NaCl}$ solution saturated with urate and spiked with $0.02 \%$ sodium azide (as bacteriostat) and were mixed overnight in a rotary mixer. Identical volumes of the seed slurries were separately added to the aliquots of the reaction medium to give a final seed suspension concentration of $6 \mathrm{mg} / 100 \mathrm{ml}$ : no seeds were added to the internal control. This instead was supplemented with an equivalent volume of $0.15 \mathrm{M} \mathrm{NaCl}$ solution saturated with urate and containing $0.02 \%$ sodium azide. The volume and size of the seed crystals added were determined by using a Coulter Counter (Model TA II) fitted with a Population Count Accessory and a $70 \mu \mathrm{m}$ orifice. Crystallization of $\mathrm{CaOx}$ was then induced in the samples by the dropwise addition of a solution of sodium oxalate $(20 \mathrm{mM})$, such that its final concentration in the samples was $0.2 \mathrm{mM}$. The samples were incubated for $120 \mathrm{~min}$ in a shaking water bath at $37^{\circ} \mathrm{C}$, and the size distributions of the suspended particles were determined at 15 min intervals using the Coulter Counter. Preliminary experiments revealed that the intra- and interassay coefficients of variation of determination of modal particle size were 6.1 and $8.9 \%$ respectively. Each experiment was performed in triplicate. These samples will be referred to as "cold."

Measurement of the Mineral Deposition by ${ }^{14} \mathrm{C}$-oxalate The use of the Coulter Counter to measure particle volume in the type of experiment described here has well documented limitations that have essentially been described earlier (27). In summary, the Coulter Counter measures particles whose sizes fall within a specified range (in these experiments 2-25.4 $\mu \mathrm{m}$ ); crystals whose sizes lie outside this range will not be counted. Furthermore, loose aggregates of crystals containing empty spaces are recorded by the instrument as if they are solid, thereby giving erroneously high estimates of particle volume deposition. This is further compounded by the inclusion of macromolecules into the crystalline architecture. Finally, the Coulter Counter cannot account for differences in particle density. Therefore, to determine the true extent of mineral deposition, parallel incubations were carried out with samples containing ${ }^{14} \mathrm{C}$ oxalate $(3.125 \mu \mathrm{Ci} / 100 \mathrm{ml})$, in which any alterations in radioactivity must reflect corresponding changes in $\mathrm{CaOx}$ precipitation. Radioactive samples were treated identically to those described above, except that they were supplemented with ${ }^{14} \mathrm{C}$-oxalic acid before the addition of sodium oxalate solution to induce crystallization.

At intervals of $15 \mathrm{~min}, 1 \mathrm{ml}$ of each sample was filtered $(0.22 \mu \mathrm{m})$ into $100 \mu \mathrm{l}$ of concentrated $\mathrm{HCl}$ using disposable syringes fitted with filters (Sartorius Minisart NML, Gottingen, Germany). Duplicate $0.3 \mathrm{ml}$ aliquots of these solutions were added to $10 \mathrm{ml}$ of Ready Safe scintillation fluid (Beckman Instruments Inc., USA) and counted for $5 \mathrm{~min}$ in a liquid scintillation counter (Beckman LS 3801 Liquid Scintillation System). Preliminary experiments revealed that the intra- and inter-assay coefficients of variation of measurement of $\mathrm{CaOx}$ deposition by ${ }^{14} \mathrm{C}$-oxalate analysis were 3.9 and $5.1 \%$ respectively. Each experiment was performed in triplicate. These samples will be referred to as "hot."

\section{Scanning Electron Microscopy (SEM)}

At the end of each experiment, $2 \mathrm{ml}$ aliquots of each cold sample were filtered $(0.22 \mu \mathrm{m})$ and the filtration membranes were dried overnight at $37^{\circ} \mathrm{C}$. They were mounted on aluminium stubs, spluttered with gold for 180 seconds (SEM Autocoating Unit E5200, Polaron Equipment Ltd, Watford, UK), and examined using an ETEC Auto Scan Electron Microscope (Siemens AG, Karlsruhe, Germany) at an operating voltage of $20 \mathrm{kV}$.

\section{Statistical Methods}

For the sake of clarity, data were plotted as mean values: nonetheless, statistical comparisons were performed using the Wilcoxon signed rank sum test at a 0.05 level of significance.

\section{Protein Analysis of Preincubated Seed Crystals} of $U A, \mathrm{NaU}$, and $\mathrm{CaOx}$

Demineralization of the Seed Crystals Treated seed crystals of UA, NaU, and $\mathrm{CaOx}, 1.5 \mathrm{~g}$ each, were separately ground to a fine powder in an agate mortar. While the CaOx crystals were demineralized using 0.25 M EDTA (pH 8.0), as described earlier (29), slurries of the UA and NaU seeds were made in $100 \mathrm{ml}$ of $0.025 \mathrm{M}$ Tris- $\mathrm{HCl}$ containing $0.02 \%$ sodium azide. They were separately transferred to dialysis bags (with nominal molecular weight cutoff of $10 \mathrm{kDa}$ ) that were stirred at $4^{\circ} \mathrm{C}$ in cold room in a conical glass flask containing 6 litre of the same buffer as used for making their slurries. To ensure complete mixing, the dialysis bags were gently mixed and their polarities reversed every 12 hours. The dialyzate was changed every 24 hours. The resulting extracts of the crystals were applied to a Bio-Gel P-6 DG desalting column. The protein peaks were collected, lyophilized, and stored at $-20^{\circ} \mathrm{C}$ until further use. 
Sodium Dodecylsulphate Polyacrylamide Gel Electrophoresis (SDS-PAGE) All the protein samples were reduced with 2-mercaptoethanol and electrophoresed in $1 \mathrm{~mm}$ thick, 9-18\% linear gradient gel on a Bio-Rad MiniProtean II apparatus (Bio-Rad Laboratories, Hercules, CA, USA), as described previously (29). The gel was subsequently stained with silver (30).

\section{Results}

\section{Particle Volumes and Sizes of Untreated and Treated} Seed Crystals

Figure 1 shows the volume distributions of untreated and treated seed crystals at zero time, ie., before the addition of oxalate solution to induce crystallization. Table 1 summarizes Figure 1. Several features of the data are noteworthy: 1) Treated or untreated, the average total particle volumes of $\sim 13,000-15,000 \mu \mathrm{m}^{3} / \mu \mathrm{l}$ of $\mathrm{NaU}$ and $\mathrm{CaOx}$ seed crystals were significantly smaller than the corresponding value of $\sim 21,000$ $\mu \mathrm{m}^{3} / \mu$ l obtained with UA seeds. The ratio of added seed crystal mass to volume of the incubation solution was identical in all cases. 2) The average total particle volumes of untreated seed crystals were very similar to those of their treated counterparts: $21,125,13,141$ and $14,768 \mu \mathrm{m}^{3} / \mu$ l for untreated and $21,350,12,810$, and $15,329 \mu \mathrm{m}^{3} / \mu \mathrm{l}$ for treated seed crystals of $\mathrm{UA}, \mathrm{NaU}$, and $\mathrm{CaOx}$, respectively. 3) Treated or untreated, the average modal particle sizes of 2.9-3.5 $\mu \mathrm{m}$ of $\mathrm{NaU}$ and $\mathrm{CaOx}$ seed crystals were significantly smaller than the corresponding value of $\sim 8.1 \mu \mathrm{m}$ obtained with UA seeds. 4) The average modal particle sizes of untreated and treated seed crystals were almost indistinguishable. 8.2, 3.0, and $3.3 \mu \mathrm{m}$ for untreated and 8.0, 2.9, and $3.5 \mu \mathrm{m}$ for treated seed crystals of $\mathrm{UA}, \mathrm{NaU}$, and $\mathrm{CaOx}$, respectively. The difference in average total particle volume and average modal particle size of seeds of different types, could perhaps be attributed to their chemical composition, which in turn, determines
Table 1. The average total particle volumes and average modal particle sizes of untreated and treated seed crystals of $\mathrm{UA}, \mathrm{NaU}$, and $\mathrm{CaOx}$

\begin{tabular}{lcc}
\hline Seed Type & $\begin{array}{c}\text { Average Total } \\
\text { Particle Volume } \\
\left(\mu \mathrm{m}^{3} / \mu \mathrm{l}\right)\end{array}$ & $\begin{array}{c}\text { Average Modal } \\
\text { Particle Size } \\
(\mu \mathrm{m})\end{array}$ \\
\hline UA untreated & 21,125 & 8.2 \\
UA treated & 21,350 & 8.0 \\
NaU untreated & 13,141 & 3.0 \\
NaU treated & 12,810 & 2.9 \\
CaOx untreated & 14,768 & 3.3 \\
CaOx treated & 15,329 & 3.5 \\
\hline
\end{tabular}

their size, degree of aggregation, and density. The shape of the volume distribution curves indicate that a significant proportion of $\mathrm{CaOx}$ and more so of $\mathrm{NaU}$ seeds, both untreated and treated, lay below the $2 \mu \mathrm{m}$ detection limit of the Coulter Counter. As noted previously (27), this will introduce an error in the estimation of total particle volume deposited after the addition of oxalate solution to induce crystallization, because $\mathrm{CaOx}$ crystals deposited in response to the oxalate solution form composite particles with seed crystals. This causes the seeds which are below the detection limit of the Coulter Counter to be detected and recorded in the higher size channel than they would have been had they not been attached to the $\mathrm{CaOx}$ crystals. The net increase in total particle volume recorded at the end of the incubation period must represent an overestimate of the increase in total particle volume in the presence of $\mathrm{CaOx}$ and $\mathrm{NaU}$ seeds, unlike the estimates obtained in the presence of UA seed crystals. For all practical purposes, Figure 1 reveals that the average modal particle sizes of all the types of seed crystals were detected by the Coulter Counter.

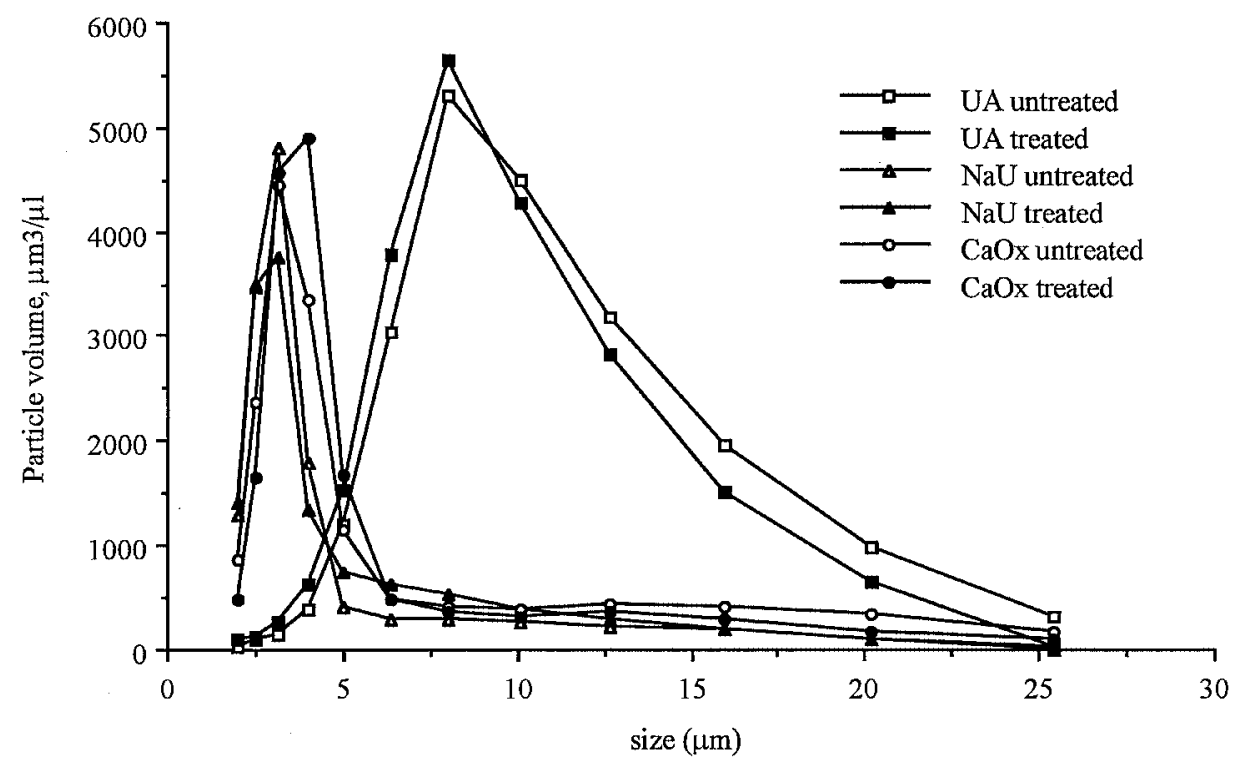

Fig. 1. Particle size distribution of untreated and treated seed crystals of uric acid (UA), sodium urate (NaU) and calcium oxalate (CaOx). This shows that the average modal particle sizes of each seed suspension were within the Coulter Counter specified limits (in these experiments, 2-25.4 $\mu \mathrm{m}$ ). 


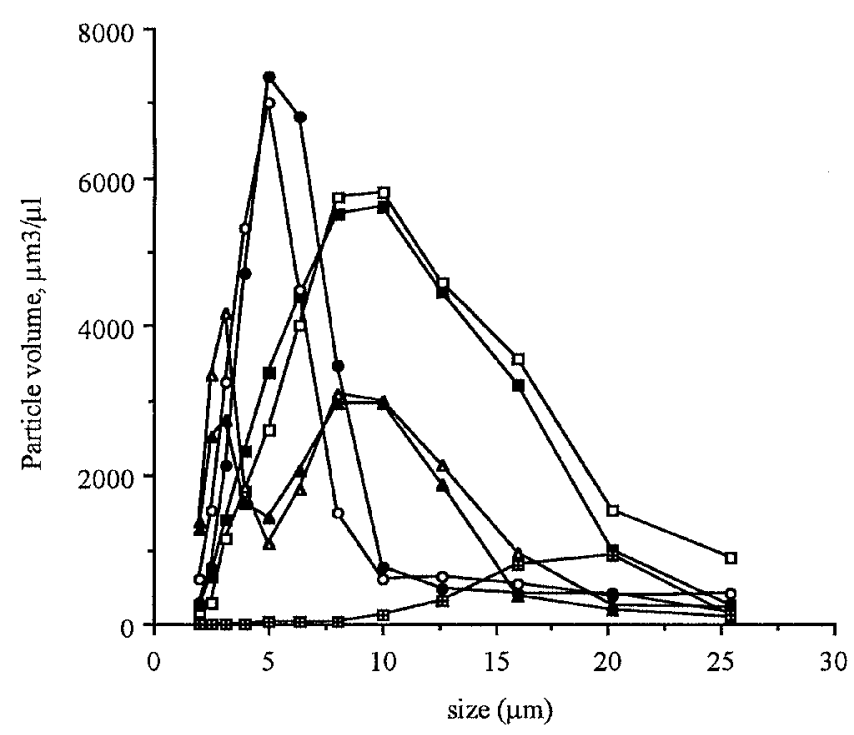

The Effect of Untreated and Treated Seed Crystals on the Deposition of Particle Size

Figure 2 shows the volume distribution of particles after $2 \mathrm{~h}$ incubation in the internal control and in aliquots of the same incubation solution which were separately supplemented with untreated and treated seed crystals of $\mathrm{UA}, \mathrm{NaU}$, and $\mathrm{CaOx}$. This figure has also been summarized in Table 2 . The data have not been corrected for the volume of the added seed crystals occurring within each size range at zero time. Correction of the data in each channel to account for those in the zero time control would have caused inaccuracies in the volume distribution curves: these curves simply show the relative sizes of the precipitated particles. The data reveal that the average modal size of the particles deposited in internal control, containing no added seeds, is $19.0 \mu \mathrm{m}$. This value is significantly reduced to varying extents by the addition of untreated and treated seed crystals of different chemical composition: 8.8, 2.9, and 8.9,

Table 2. The uncorrected average modal sizes of the particles deposited 2 hours after the addition of oxalate solution to the internal control (no seeds) and portions of the same incubation solution that were separately supplemented with untreated and treated seed crystals of $\mathrm{UA}, \mathrm{NaU}$, and $\mathrm{CaOx}$

\section{Sample}

UA untreated

UA treated

NaU untreated

NaU treated

CaOx untreated

CaOx treated

Internal control
Uncorrected Average Modal Particle Size $(\mu \mathrm{m})$

8.8

9.0

2.9 and 8.9

2.9 and 9.1

5.0

5.3

19.0
Fig. 2. Particle size distribution at 2 hours after the addition of oxalate solution to the internal control (no seeds) and portions of the same incubation solution which were separately spiked with untreated and treated seed crystals of $\mathrm{UA}, \mathrm{NaU}$ and CaOx. The values represent that (a) the average modal sizes of particles precipitated in the presence of treated seed crystals of $\mathrm{UA}, \mathrm{NaU}$, and $\mathrm{CaOx}$ were very similar to those deposited in the presence of their respective untreated controls (b) irrespective of whether or not seed crystals of UA and NaU had been treated, the average modal sizes of particles precipitated in their presence were at least half relative to those deposited in internal control, containing no added seeds.

$5.0 \mu \mathrm{m}$ in the presence of untreated and 9.0, 2.9, and 9.1, $5.3 \mu \mathrm{m}$ in the presence of treated seed crystals of $\mathrm{UA}, \mathrm{NaU}$, and $\mathrm{CaOx}$, respectively. The average modal sizes of the particles were increased, in sequence, by 2.7 (non-significant), 1.2 (non-significant), and 2.5 (non-significant), 5.7\% (non-significant) in the presence of treated seed crystals in comparison with their respective untreated controls.

The volume distribution curves of the incubations spiked with untreated and treated NaU seeds are bimodal, most likely because the newly precipitated CaOx crystals form composite particles with the seed crystals and are recorded in the higher size channel. Since the number of NaU seed particles in the incubations is very high $(143,576$ for untreated and 138,143 for treated) as compared with the number of $\mathrm{CaOx}$ particles deposited (which in internal control, containing no added seeds, was 864), a large proportion of the seed crystals would have remained unattached to the freshly deposited CaOx. The NaU seeds alone would be recorded at lower size channel while the ones attached with newly precipitated $\mathrm{CaOx}$ crystals would be recorded at higher size channel. The presence of these two types of particle populations in these incubations explains their bi-modal distribution. At the end of the 2 hour incubation the average lower modal value (of the bi-modal distributions) obtained in samples containing untreated and treated NaU seeds is $2.9 \mu \mathrm{m}$. This value is almost identical to the average modal size value of $2.95 \mu \mathrm{m}$ of untreated and treated NaU seed particles alone, as recorded by the Coulter Counter at 0 min (Fig. 3).

Figure 3 shows low power scanning electron micrographs of the particles deposited at the end of the 2 hour incubation period in the internal control and in portions of the same incubation solution which were separately spiked with untreated and treated seed crystals of UA, NaU, and CaOx. The crystals deposited in all the incubations were principally coffin shaped $\mathrm{CaOx}$ monohydrates, which in internal 
Induction of CaOx crystallization by sood crystals: Effect of preincubatien with urine

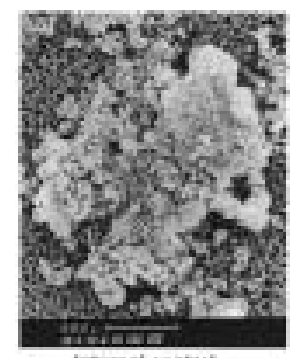

Iraned cortril
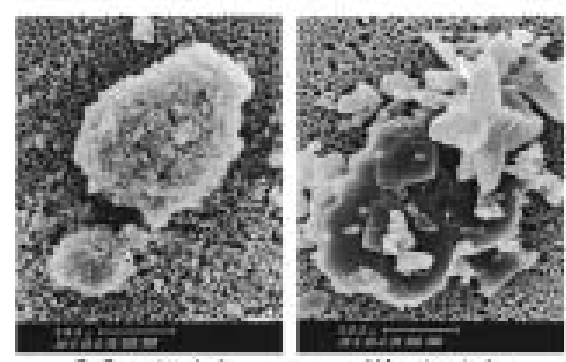
UA urtrealad

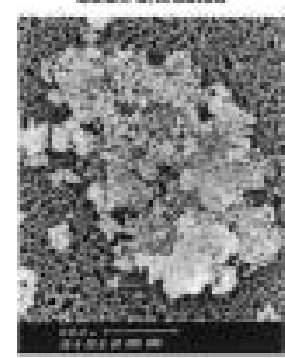

CeOr treoted

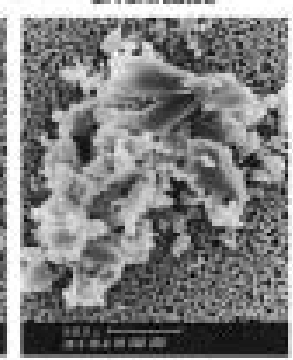

Lh rating

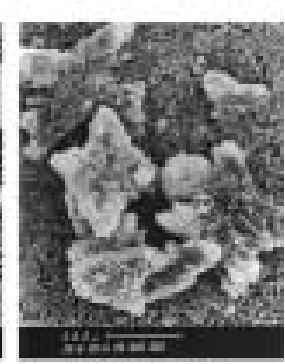
NaU untwoinas

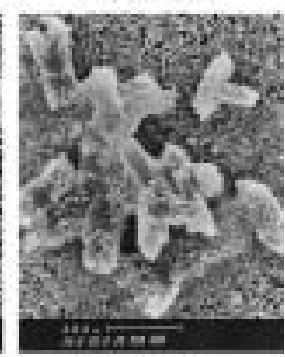

Now trentind

Fig. 3. Low-power scanning electron micrographs of the crystalline material deposited 2 hours after the addition of oxalate solution to internal control, containing no added seeds, and samples of the same incubation solution which were separately supplemented with untreated and treated seed crystals of $\mathrm{UA}, \mathrm{NaU}$, and $\mathrm{CaOx}$. This shows that (a) the sizes of particles precipitated in the presence of treated seed crystals of UA, NaU, and CaOx were very similar to those deposited in the presence of their respective untreated controls (b) irrespective of whether or not seed crystals of UA and $\mathrm{NaU}$ had been treated, some of them were seen lying free on the filtration membrane while others were attached like barnacles onto the surfaces of the CaOx crystals.

control, containing no added seeds, were small and loosely grouped to form large clusters. Likewise, the individual $\mathrm{CaOx}$ crystals precipitated in the presence of untreated and treated CaOx seeds were also very small and their degree of aggregation in the former was seemingly higher than in the latter. Similarly, the degree of particle aggregation in the incubations containing untreated UA and NaU seeds was apparently higher than in the incubations containing their treated controls. In sharp contrast, however, the individual $\mathrm{CaOx}$ crystals deposited in the presence of UA and NaU seeds, both untreated and treated, were quite large as compared with those precipitated in the internal control. The scanning electron micrographs also revealed that whether or not the seeds

had been treated, the size of individual seed crystals of UA is an order of magnitude larger as compared with those of NaU. This confirms the observation of large particles of seed crystals of UA relative to those of $\mathrm{NaU}$, as revealed by the Coulter Counter at $0 \mathrm{~min}$. Treated or untreated, the presence of $\mathrm{NaU}$, and, to a lesser extent, UA seeds was noted on the filter membrane. Also, the seed crystals of UA and NaU, both untreated and treated, can be seen attached like barnacles upon the surfaces of precipitated $\mathrm{CaOx}$ crystals.

\section{The Effect of Untreated and Treated Seed Crystals on the Deposition of Calcium Oxalate as Determined by ${ }^{14} \mathrm{C}$-oxalate Analysis}

Figure 4 and Table 3 show the time course of the disappearance of ${ }^{14} \mathrm{C}$-oxalate during the 2 hour incubation period following the addition of oxalate solution to the internal control and to portions of the same incubation solution which were separately spiked with untreated and treated seed crystals of UA, $\mathrm{NaU}$, and $\mathrm{CaOx}$. To normalize the data the values were presented as per cent ${ }^{14} \mathrm{C}$-oxalate remaining in the solution relative to that recorded in each sample at zero time. The rate of reduction in ${ }^{14} \mathrm{C}$-oxalate was either the same or greater in all the incubations supplemented with untreated or treated seed crystals than in internal control to which no seeds had been added. Irrespective of whether or not CaOx seed crystals had been treated, the values recorded in the incubations differed dramatically from those obtained in the presence of UA and NaU seeds. At 2 hour the mean percentage reduction in ${ }^{14} \mathrm{C}$-oxalate in the internal control $(64.7 \%)$ was very similar to the value obtained with untreated UA seed crystals (65.9\%). However, this value was significantly reduced to 40.8 and $9.8 \%$ following the addition of untreated seed crystals of $\mathrm{NaU}$ and $\mathrm{CaOx}$, respectively. This represented that while untreated UA seeds inhibited the precipitation of $\mathrm{CaOx}$ by $1.9 \%$ (non-significant), untreated $\mathrm{NaU}$ and CaOx seeds promoted the process by $36.9(p \leq$ $0.05)$ and $84.8 \%(p \leq 0.05)$ respectively relative to internal control, containing no added seeds.

The corresponding values of percentage reduction in ${ }^{14} \mathrm{C}$-oxalate obtained following the addition of treated seed crystals of UA, NaU, and CaOx were, in sequence, $65.7,61.9$ and $10.7 \%$. It therefore follows that while treated seed crystals of UA caused a very small promotion in the deposition of $\mathrm{CaOx}$ by $0.3 \%$ (non-significant), treated NaU and CaOx seeds inhibited the same process by $51.9(p \leq 0.05)$ and $8.5 \%$ $(p \leq 0.05)$ respectively in comparison with the incubations containing their respective untreated controls.

Figure 5 shows SDS-PAGE of proteins of the urine sample in which UA, NaU, and $\mathrm{CaOx}$ seed crystals were preincubated, and of the demineralised extracts of those crystals. As always, the urine contained a very large spectrum of proteins. However, only a handful of them were seen in the extracts of treated seed crystals. The electrophoretogram 


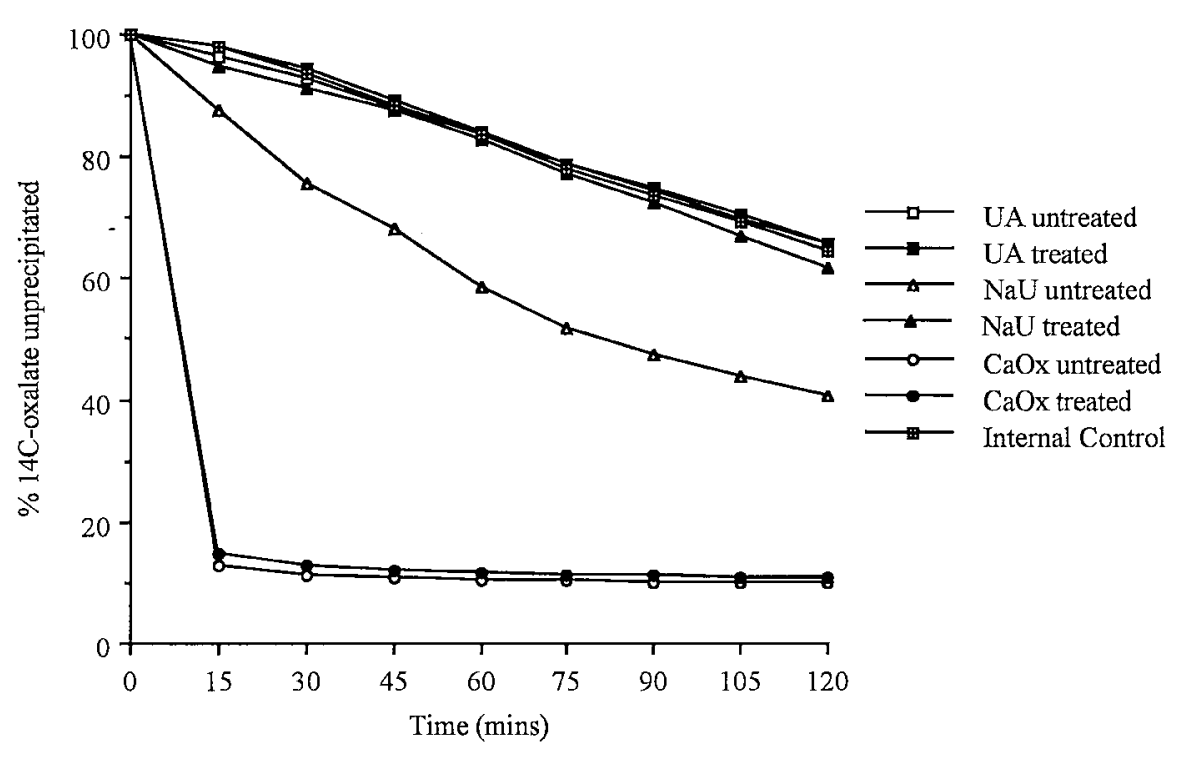

Fig. 4. Change in unprecipitated ${ }^{14} \mathrm{C}$ oxalate during $2 \mathrm{~h}$ incubation after the addition of oxalate solution to the internal control (no seeds), and aliquots of the same incubation solution which were separately spiked with untreated and treated seed crystals of $\mathrm{UA}, \mathrm{NaU}$, and $\mathrm{CaOx}$. The values obtained represent that while untreated seed crystals of UA did not alter the deposition of CaOx, those of $\mathrm{NaU}$ and $\mathrm{CaOx}$ significantly promoted the process by 36.9 and $84.8 \%$ respectively in comparison with internal control to which no seeds were added. The results also showed that preincubation of the seed crystals with urine, such as occurs in vivo, only slightly reduced the ability of seed crystals of $\mathrm{CaOx}$, but not of UA, to cause the mineral deposition in comparison with their respective untreated controls. The most dramatic effect was seen with NaU seed crystals where the preincubation inhibited by $51.9 \%$ their ability to promote deposition of $\mathrm{CaOx}$ relative to incubations to which untreated NaU seed crystals were added. also showed that the number and molecular weights of proteins adsorbed onto the preincubated seed crystals varied depending upon chemical composition of the seeds. It is remarkable that a protein with molecular weight of approximately $31 \mathrm{kDa}$ was predominant in the extract of treated $\mathrm{CaOx}$ seeds. Previously known as crystal matrix protein, it has now been identified as urinary form of prothrombin fragment 1 (31-32). In this investigation, no attempt was made to identify proteins in the extracts of treated UA and NaU seed crystals. For all practical purposes Fig. 5 reveals that seed crystals preincubated with urine adsorbed proteins onto their crystal surface.

\section{Discussion}

Almost 30 years ago, Dent and Sutor (33) made an empirical clinical observation that "hyperuricosuria" seemed to be more common in stone formers than in normal subjects. Since then a great deal of effort has been made to clarify the association of urinary urate excretion with $\mathrm{CaOx}$ stone formation. Perhaps the most powerful evidence to support a determinant role for urate has been reports that allopurinol appears to reduce $\mathrm{CaOx}$ stone recurrences in patients whose only detectable abnormality is hyperuricosuria (4-11). A number of mechanisms were proposed in an attempt to explain this apparent beneficial effect of allopurinol. Of these, epitaxy has been the most often cited, its

Table 3. Change in unprecipitated ${ }^{14} \mathrm{C}$-oxalate during 2 hour incubation after the addition of oxalate solution to internal control, to which no seed crystals were added, and aliquots of the same incubation solution that were separately spiked with untreated and treated seed crystals of $\mathrm{UA}, \mathrm{NaU}$, and $\mathrm{CaOx}$

\begin{tabular}{|c|c|c|c|c|c|c|c|}
\hline \multirow[b]{2}{*}{$\begin{array}{l}\text { Time } \\
\text { (min) }\end{array}$} & \multicolumn{7}{|c|}{ Change in Precipitation (\%) } \\
\hline & $\begin{array}{l}\text { Internal } \\
\text { Control }\end{array}$ & $\begin{array}{c}\text { UA } \\
\text { Untreated }\end{array}$ & $\begin{array}{c}\text { UA } \\
\text { Treated }\end{array}$ & $\begin{array}{c}\text { NaU } \\
\text { Untreated }\end{array}$ & $\begin{array}{c}\text { NaU } \\
\text { Treated }\end{array}$ & $\begin{array}{c}\text { CaOx } \\
\text { Untreated }\end{array}$ & $\begin{array}{c}\text { CaOx } \\
\text { Treated }\end{array}$ \\
\hline 0 & 100 & 100 & 100 & 100 & 100 & 100 & 100 \\
\hline 15 & 98.0 & 96.5 & 98.1 & 87.5 & 95.0 & 12.7 & 14.7 \\
\hline 30 & 93.5 & 92.6 & 94.3 & 75.8 & 91.3 & 11.3 & 12.5 \\
\hline 45 & 88.3 & 88.1 & 89.1 & 67.9 & 87.5 & 10.7 & 11.9 \\
\hline 60 & 83.5 & 83.4 & 84.0 & 58.6 & 82.7 & 10.3 & 11.4 \\
\hline 75 & 78.1 & 78.7 & 79.0 & 51.8 & 77.3 & 10.3 & 11.1 \\
\hline 90 & 73.7 & 74.5 & 74.9 & 47.3 & 72.5 & 10.0 & 11.0 \\
\hline 105 & 69.4 & 69.7 & 70.5 & 44.0 & 66.9 & 9.9 & 10.8 \\
\hline 120 & 64.7 & 65.9 & 65.7 & 40.8 & 61.9 & 9.8 & 10.7 \\
\hline
\end{tabular}




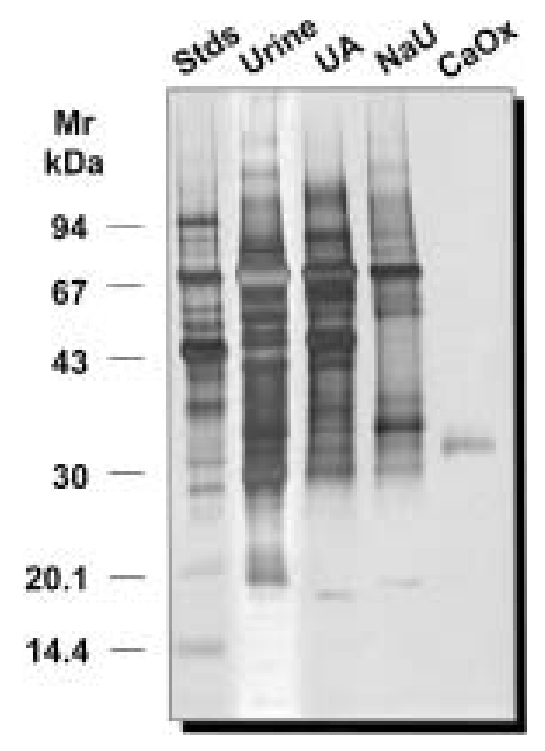

Fig. 5. SDS-PAGE of proteins of the urine sample in which $\mathrm{UA}, \mathrm{NaU}$, and CaOx seed crystals were preincubated, and of the demineralized extracts of those crystals. Note that while the urine contained a very large spectrum of proteins only a handful of them were seen in the extracts of treated seed crystals. The presence of a protein with molecular weight of approximately $31 \mathrm{kDa}$ was remarkable in the extract of treated $\mathrm{CaOx}$ seeds. Previously known as crystal matrix protein, it has now been identified as urinary form of prothrombin fragment 1 .

credibility having been reinforced by in vitro studies demonstrating that crystalline particles of $\mathrm{NaU}$, and to a lesser extent UA, can induce precipitation of $\mathrm{CaOx}$ from metastable solutions of this salt (15-26,34-35). The theoretical aspects of this theory were already bolstered by X-ray crystallographic data revealing the existence of several crystal lattice fits for UA, UA dihydrate, whewellite (CaOx monohydrate), and weddellite (CaOx dihydrate) (14). Based on these theoretical and experimental evidence the theory could successfully explain (a) the formation of kidney stones consisting of mixtures of different minerals (b) a link between hyperuricosuria and $\mathrm{CaOx}$ stone formation (c) success of allopurinol in reducing $\mathrm{CaOx}$ stone recurrence. Therefore, it is not surprising that epitaxy has been the most commonly proposed explanation for clinical observations connecting urate and $\mathrm{CaOx}$ stone disease and the justification for therapeutic use of allopurinol. However, as far as the actual formation of stones is concerned, the scientific evidence on which this theory is largely based is physiologically irrelevant. This is mainly because stones are formed in real urine and not in inorganic solutions as used in these studies. The physicochemical characteristics of urine are quite different from that of synthetic inorganic solutions of $\mathrm{CaOx}$ even when designed to mimic urine as close as possible. This cautions against extrapolating findings from inorganic solutions to predict likely effects in whole urine, particularly on stone formation. Also, it is now widely accepted that urine contains large numbers and quantities of low and high molecular weight components, some of which are well-documented inhibitors of CaOx crystallization (36). Furthermore, the concentrations and relative amounts of various urinary components vary from one urine specimen to another. Thus it is fair to say that the effects of all urinary components on the epitaxial relationship between urate and $\mathrm{CaOx}$ cannot possibly be reproduced under experimental conditions using inorganic salt solutions in vitro.

Recently, we investigated the effect of seed crystals of UA and $\mathrm{NaU}$ on crystallization of CaOx in undiluted human urine in vitro (27). The results demonstrated conclusively that these seed crystals do not promote $\mathrm{CaOx}$ deposition to a physiologically significant degree. In the present investigation an attempt was made to clarify whether the lack of promotory effect of urate seeds on CaOx crystallization in undiluted urine could be attributed to the "surface poisoning" of seed crystals, presumably the result of adsorption of urinary macromolecules and other low molecular weight components. This was achieved by comparing the ability of UA and NaU seeds which were preincubated with urine, with their untreated controls to induce precipitation of $\mathrm{CaOx}$ in synthetic inorganic solution as the reaction medium.

Although the use of whole urine would have been ideal, only centrifuged and $0.22 \mu \mathrm{m}$ filtered urine was used for preincubating seed crystals. This was because urine invariably contains particulate material (37) that after preincubation with seed crystals would be practically impossible to remove. It is remarkable that centrifugation and $0.22 \mu \mathrm{m}$ filtration of urine is known to remove Tamm-Horsfall glycoprotein and some human serum albumin (37) - neither of which has any significant effect on CaOx crystal growth in undiluted human urine (38). With these reservations, commercial seed crystals of UA, NaU, and $\mathrm{CaOx}$ were mixed separately with aliquots of the same centrifuged and $0.22 \mu \mathrm{m}$ filtered urine at an identical ratio of added seed crystal mass to urine volume of $1 \mathrm{~g} / \mathrm{l}$ each. The samples were stirred for 3 hours at room temperature using magnetic stir bar in conical glass flasks. The crystals were harvested by filtration through $0.22 \mu \mathrm{m}$ Millipore filters, washed thoroughly with distilled water and lyophilized. The control (or untreated) seed crystals consisted of the same commercial batch of seeds of $\mathrm{UA}, \mathrm{NaU}$, and $\mathrm{CaOx}$ which had not been preincubated with urine.

Initial attempts were made to study the effect of untreated and treated seed crystals on CaOx crystallization in a reaction medium as described by Pak and Arnold (16). The final suspension concentration of each type of seed crystals used was $6 \mathrm{mg} / 100 \mathrm{ml}$. All the samples including internal control, to which no seed crystals were added, were incubated in a shaking water-bath at $37^{\circ} \mathrm{C}$ : untreated and treated seed crystals of UA and NaU dissolved within 15-30 minutes. Similar dissolution was noted using the reaction medium as described by Coe et al. (15). Therefore, the effects of the seed crystals on CaOx 
crystallization were studied in a modified solution which had the same calcium and oxalate concentrations as used by Coe et al. (15), but in addition was also saturated with commercial seed crystals of UA and $\mathrm{NaU}$. It is interesting to note that the reaction media similar to the ones described by Coe et al. (15) and Pak and Arnold (16) were used by various investigators who used vastly different techniques to examine the effect of UA and NaU seeds on $\mathrm{CaOx}$ crystallization. However, they made no mention of dissolution of the seed crystals except some $(17,24,39-40)$ who then saturated incubation media, as in the present investigation, with commercial seed crystals of UA and/or NaU. Analysis of ${ }^{14} \mathrm{C}$-oxalate data revealed that while untreated seed crystals of UA did not alter the deposition of $\mathrm{CaOx}$, those of $\mathrm{NaU}$ and $\mathrm{CaOx}$ significantly promoted the process by 36.9 and $84.8 \%$ respectively in comparison with internal control to which no seeds were added. While these results are in perfect agreement with those of several previous studies (15-17,23,34-35) suggesting that NaU, but not UA, promote deposition of $\mathrm{CaOx}$ from its aqueous inorganic solutions, they are contradictory to others $(18,20-21,23,25-26)$ who found even UA to be an effective promoter of the process as well. The results also showed that preincubation of the seed crystals with urine, such as occurs in vivo, only slightly reduced the ability of seed crystals of $\mathrm{CaOx}$, but not of UA, to cause the mineral deposition in comparison with their respective untreated controls. The most dramatic effect was seen with NaU seed crystals where the preincubation inhibited by $51.9 \%$ their ability to promote deposition of $\mathrm{CaOx}$ relative to incubations to which untreated NaU seed crystals were added.

The size of the crystalline particles precipitated in the kidney tubules is an important determinant of their likelihood of retention within the renal collecting system and hence, of stone formation. The results of this study revealed that all untreated seeds tested reduced particle size. The average modal size of the particles deposited in the internal control, $19.0 \mu \mathrm{m}$, was reduced significantly to $8.8,2.9$, and $8.9,5.0 \mu \mathrm{m}$ in the presence of added untreated seed crystals of UA, NaU, and $\mathrm{CaOx}$ respectively. Thus the average modal size of the particles precipitated were at least $52.63 \%$ smaller in the presence of untreated UA and NaU seed crystals as compared with the internal control. No previous study has examined the effect of untreated UA and $\mathrm{NaU}$ seed crystals on the size of particles precipitated from aqueous inorganic solutions of $\mathrm{CaOx}$. The results of the present study also revealed that although average modal sizes of the particles precipitated with treated seed crystals of $\mathrm{UA}, \mathrm{NaU}$ and $\mathrm{CaOx}$ were slightly smaller than those deposited in the presence of their untreated controls, this difference did not achieve statistical significance. These results were confirmed empirically by scanning electron microscopy (SEM), which also revealed that whether or not UA and $\mathrm{NaU}$ seed crystals had been preincubated, they were clearly visible. Although some of them were lying free on the filtration membrane, others were attached like barnacles onto the surfaces of the CaOx crystals. One might therefore argue that had epitaxy occurred to any significant extent, UA and NaU seeds should have been overgrown with $\mathrm{CaOx}$ (thereby exhibiting the morphology of pure $\mathrm{CaOx}$ ) or be surrounded by newly nucleated $\mathrm{CaOx}$ crystals so that they themselves would be hidden. Certainly, this was not the case with untreated and treated UA and NaU seeds. This might seem to militate against credibility of the epitaxy theory. However, the ${ }^{14} \mathrm{C}$-oxalate data revealed that untreated seed crystals of $\mathrm{NaU}$, but not UA, did promote precipitation of $\mathrm{CaOx}$ by $36.9 \%$, as compared with internal control to which no seeds were added. This, coupled with the fact that several lattice fits between urate and $\mathrm{CaOx}$ have already been reported (14), strongly suggests that at least some, if not all, $\mathrm{CaOx}$ crystals deposited in the presence of untreated $\mathrm{NaU}$ seeds may, in fact, have NaU seed(s) as their nidi. A direct confirmation of this is difficult because it is practically impossible to separate $\mathrm{NaU}$ seeds from the freshly precipitated CaOx crystals. This raises an unanswered question: why only some, and not all, untreated NaU seeds act as nidi for the deposition of CaOx. The SEM observations of the present investigation are in accordance with previous studies where UA and/or $\mathrm{NaU}$ seeds promoted the mineral deposition and were themselves clearly visible attached to the freshly precipitated CaOx $(17-18,21,25-26,40)$. The scanning electron microscopy results of this study also revealed that irrespective of whether or not UA and $\mathrm{NaU}$ seeds had been preincubated, the individual $\mathrm{CaOx}$ crystals deposited in their presence were quite large. This is consistent with their enhanced crystal growth-perhaps the result of their decreased crystal aggregation due to attachment like barnacles of UA and more so of NaU seeds onto the surface of freshly deposited CaOx crystals. This supposition is supported by the Coulter Counter data that at the end of $2 \mathrm{hr}$ incubation the average modal sizes of precipitated particles in the presence of untreated and treated seed crystals of UA and NaU were almost half to that deposited in the internal control containing no added seeds. More importantly, the results suggest that UA and NaU seeds with adsorbed urinary macromolecules, as would happen in urine in vivo, decrease crystal aggregation and thus would tend to reduce the likelihood of stone formation, as this the only process which, even in the absence of any crystal growth, can result in the formation of large, potentially dangerous particles in a short span of time.

The results of protein analyses of treated seed crystals revealed that they all adsorbed proteins which differed in number and molecular weight depending upon chemical composition of the seeds. This confirms the widely acknowledged view that seed crystals bind urinary proteins in a selective manner (28). Previous studies have also shown similar protein profiles in extracts of demineralized UA and $\mathrm{CaOx}$ crystals generated in human urine $(29,41-42)$. It is remarkable that 
urinary form of prothrombin fragment 1 (UPTFl) is the predominant protein adsorbed onto the surface of treated $\mathrm{CaOx}$ seed crystals. And amongst the macromolecules it has the most inhibitory effect on $\mathrm{CaOx}$ crystallization thus far tested in undiluted human urine in vitro (43). This strong inhibitory effect of UPTFl, like its parent molecule prothrombin, is almost certainly due to the ten $\gamma$-carboxyglutamic acid (Gla) residues in its $\mathrm{N}$-terminal region, which define its Gla domain (44). Gla, which is a relatively uncommon amino acid occurs principally in proteins involved in calcification or blood coagulation, is formed by vitamin-K-dependent post-translational $\gamma$-carboxylation of glutamic acid residues, and imparts an exceptional $\mathrm{Ca}^{2+}$-binding capacity to proteins in which it is found (45). Thus despite the perfect lattice match, slight attenuation of the ability of treated $\mathrm{CaOx}$ seed crystals to induce deposition of $\mathrm{CaOx}$ observed in this study may perhaps be attributable to the adsorption of UPTF1 onto the surface of preincubated CaOx seeds. It is interesting to note that though in the present investigation untreated and treated UA seeds did not effect precipitation of $\mathrm{CaOx}$ what so ever, proteins of peaks I-III obtained after DEAE-cellulose column chromatography of demineralized extracts of their crystals generated in urine of healthy subjects have recently been reported to inhibit the nucleation of $\mathrm{CaOx}$ (46). Collectively, the findings suggest that the slight attenuation of the ability of the treated seed crystals of $\mathrm{CaOx}$ and more importantly of $\mathrm{NaU}$ to cause deposition of $\mathrm{CaOx}$ could be attributable to the binding of proteins, and perhaps other urinary macromolecules and low molecular weight components (28) onto the surface of seed crystals following their preincubation with urine.

Taken together, results of the present study indicate that untreated seed crystals of $\mathrm{NaU}$, but not of UA, promote deposition of $\mathrm{CaOx}$ from its synthetic inorganic solution. And this promotory effect of NaU seeds is attenuated quite dramatically following their preincubation with urine. This may explain the discrepancy between the findings of studies carried out in inorganic solutions and undiluted human urine and further stresses the invalidity of directly extrapolating results obtained in inorganic solutions to likely effects in urine and more importantly, on stone formation. As reported previously UA and NaU seeds do not promote $\mathrm{CaOx}$ deposition to a physiologically significant degree in urine (27). Therefore epitaxial induction of CaOx precipitation by urate seeds is unlikely to be a major factor contributing to stone formation. This view is further reinforced by the fact that the crystallization of $\mathrm{NaU}$ in urine requires excretion of large quantities of sodium and urate ions which are physiologically unattainable (47). We have previously shown that the addition of dissolved NaU to human urine promotes $\mathrm{CaOx}$ precipitation (48) and that this effect can not be attributed to depletion of glycosaminoglycans (49), as suggested by Robertson et al. (50), or to the epitaxial deposition of $\mathrm{CaOx}$ onto urate particles formed in response to an increase in urate concentration or addition of an oxalate load (51). In the absence of any other plausible explanation, the promotion of CaOx precipitation by dissolved urate is consistent with the mechanism of "salting-out" as suggested by Kallistratos and coworkers (52-53). Such a mechanism would explain the apparent beneficial effect of allopurinol administration, without the need to invoke the occurrence of seed crystals of NaU and UA in urine, the presence of which occurs only rarely.

\section{References}

1. Ryall RL, Grover PK, Marshall VR. (1991) Urate and calcium stones: picking up a drop of mercury with one's fingers? Am. J. Kidney Dis. 17: 426-430.

2. Grover PK, Ryall RL. (1994) Urate and calcium oxalate stones: from repute to rhetoric to reality. Miner. Electrolyte Metab. 20: 361-370.

3. Grover PK, Ryall RL. (1995) Urate and calcium oxalate stones: A new look at an old controversy. In: Khan SR, (ed.) Calcium Oxalate in Biological Systems. CRC Press, New York and London; 305-321.

4. Coe FL, Raisen L. (1973) Allopurinol treatment of uric-acid disorders in calcium-stone formers. Lancet 1: 129-131.

5. Coe FL, Kavalach AG. (1974) Hypercalciuria and hyperuricosuriain patients with calcium nephrolithiasis. N. Engl. J. Med. 291: 1344-1350.

6. Smith MJV, Boyce WH. (1969) Allopurinol and urolithiasis. J. Urol. 102: 750-753.

7. Pak CYC, Barilla DE, Holt K, et al. (1978) Effect of oral purine load and allopurinol on the crystallization of calcium salts in urine of patients with hyperuricosuric calcium urolithiasis. Am. J. Med. 65: 593-599.

8. Miano L, Petta S, Gallucci M. (1979) Allopurinol in the prevention of calcium oxalate renal stones. Eur. Urol. 5: 229-232.

9. Maschio G, Tessitore N, D'Angelo A, et al. (1981) Prevention of calcium nephrolithiasis with low dose thiazide, amiloride, and allopurinol. Am. J. Med. 71: 623-626.

10. Smith MJV. (1983) Placebo versus allopurinol for recurrent urinary calculi. Proc. EDTA 20: 422-426.

11. Ettinger B, Tang A, Citron JT, et al. (1986) Randomized trial of allopurinol in the prevention of calcium oxalate calculi. N. Engl. J. Med. 315: 1386-1389.

12. Royer L. (1928) Experimental research on parallel growth on mutual orientation of crystals of different species. Bull. Soc. Fr. Mineral. 51: 7-159.

13. Modlin M. (1967) The aetiology of renal stones: A new concept arising from studies in a stone-free population. Ann. $R$. Coll. Surg. Engl. (London) 40: 155-178.

14. Lonsdale K. (1968) Epitaxy as a growth factor in urinary calculi and gallstones. Nature 217: 56-58.

15. Coe FL, Lawton RN, Goldstein RB, Tembe V. (1975) Sodium urate accelerates precipitation of calcium oxalate in vitro. Proc. Soc. Exp. Biol. Med. 149: 926-929.

16. Pak CYC, Arnold LH. (1975) Heterogeneous nucleation of calcium oxalate by seeds of monosodium urate. Proc. Soc. Exp. Biol. Med. 149: 930-932.

17. Meyer JL, Bergert JH, Smith LH. (1976) The epitaxially induced crystal growth of calcium oxalate by crystalline uric acid. Invest. Urol. 14: 115-119.

18. Koutsoukos PG, Lam-Erwin CY, Nancollas GH. (1980) Epitaxial considerations in urinary stone formation. I. The urateoxalate-phosphate system. Invest. Urol. 18: 178-184.

19. Mandel NS, Mandel GS. (1981) Epitaxis between stoneforming crystals at atomic level. In: Smith LH, Robertson WG and Finlayson B (eds.) Urolithiasis: Clinical and Basic Research. Plenum Press, New York and London, 469-480.

20. Hartung R, Leskovar P, Kratzer M. (1981) Mechanisms of the influence of uric acid on the precipitation of Ca-oxalate 
crystals out of metastable solution. Croat. Chim. Acta 53: 381-388.

21. Deganello S, Coe F. (1983) Epitaxy between uric acid and whewellite: experimental verification. $N$. Jb. Miner. Mh. 6: 270-276.

22. Deganello S, Chou C. (1984) The uric acid-whewellite association in human kidney stones. Scan. Electron Microsc. II: 927-933.

23. Sarig S, Hirsch D, Garti N, Goldwasser B. (1984) An extension of the concept of epitaxial growth. J. Crystal Growth 69: 91-95.

24. Tiselius HG. (1984) Effects of sodium urate and uric acid crystals on the crystallization of calcium oxalate. Urol. Res. 12: 11-15.

25. Grases F, Conte A, Gil JJ. (1988) Simple method for the study of heterogeneous nucleation in calcium oxalate urolithiasis. Br. J. Urol. 61: 468-473.

26. Sikiric M, Babic-Ivancic V, Tonkovic M. (1997) Precipitation of calcium oxalate and calcium phosphate in the presence of uric acid. Colloids Surfaces A: Physicochem. Eng. Aspects 121: 145-150.

27. Grover PK, Ryall RL. (1997) Effect of seed crystals of uric acid and monosodium urate on the crystallization of calcium oxalate in undiluted human urine in vitro. Clin. Sci. 92: 205-213.

28. Ryall RL. (1996) Glycosaminoglycans, proteins, and stone formation: adult themes and child's play. Pediatr. Nephrol. 10: 656-666.

29. Doyle IR, Ryall RL, Marshall VR. (1991) Inclusion of proteins into calcium oxalate crystals precipitated from human urine: a highly selective phenomenon. Clin. Chem. 37: 1589-1594.

30. Heukeshoven J, Dernick R. (1985) Simplified method for silver-staining of proteins in polyacrylamide gels and the mechanism of silver staining. Electrophoresis 6: 103-112.

31. Stapleton AMF, Simpson RJ, Ryall RL. (1993) Crystal matrix protein is related to human prothrombin. Biochem. Biophys. Res. Commun. 195: 1199-1203.

32. Stapleton AMF, Ryall RL. (1995) Blood coagulation proteins and urolithiasis are linked: crystal matrix protein is the Fl activation peptide of human prothrombin. Br. J. Urol. 75: 712-719.

33. Dent CE, Sutor DJ. (1971) Presence or absence of an inhibitor of calcium oxalate crystal growth in urine of normals and stone-formers. Lancet ii: 775-778.

34. Pak CYC, Hayashi Y, Arnold LH. (1976) Heterogeneous nucleation with urate, calcium phosphate and calcium oxalate. Proc. Soc. Exp. Biol. Med. 153: 83-87.

35. Pak CYC, Holt K, Britton F, et al. (1980) Assessment of pathogenetic role of uric acid, monopotassium urate, monoammonium urate and monosodium urate in hyperuricosuric calcium oxalate nephrolithiasis. Miner. Electrolyte Metab. 4: 130-136.

36. Ryall RL. (1997) Urinary inhibitors of calcium oxalate crystallization and their potential role in stone formation. World $J$. Urol. 15: 155-164.

37. Doyle IR, Ryall RL, Marshall VR. (1989) The effect of low speed centrifugation and millipore filtration on the urinary protein content. In: Walker VR, Sutton RAL, Cameron ECB, Pak CYC, Robertson WG (eds.) Urolithiasis. New York: Plenum Press; 593-594.
38. Ryall RL, Harnett RM, Hibberd CM, et al. (1991) Effects of chondroitin sulphate, human serum albumin and TammHorsfall mucoprotein on calcium oxalate crystallization in undiluted human urine. Urol. Res. 19: 181-188.

39. Burns JR, Finlayson B. (1980) The effect of seed crystals on calcium oxalate nucleation. Invest. Urol. 18: 133-136.

40. Meyer JL. (1981) Nucleation kinetics in the calcium oxalatesodium urate monohydrate system. Invest. Urol. 19: 197-201.

41. Iwata H, Kamei O, Abe Y, et al. (1988) The organic matrix of urinary uric acid crystals. J. Urol. 139: 607-610.

42. Morse RM, Resnick MI. (1989) A study of the incorporation of urinary macromolecules onto crystals of different mineral compositions. J. Urol. 141: 641-644.

43. Ryall RL, Grover PK, Stapleton AMF. (1995) The urinary Fl activation peptide of human prothrombin is a potent inhibitor of calcium oxalate crystallization in undiluted human urine in vitro. Clin. Sci. 89: 533-541.

44. Grover PK, Ryall RL. (1999) Inhibition of calcium oxalate growth and aggregation by prothrombin and its fragments in vitro: relationship between protein structure and inhibitory activity. Eur. J. Biochem. 263: 50-56.

45. Burnier JP, Borowski M, Furie BC, Furie B. (1981) Gammacarboxyglutamic acid. Mol. Cellular Biochem. 39: 191-199.

46. Kalaiselvi P, Udayapriya KL, Selvam R. 1999) Uric acidbinding proteins in calcium oxalate stone formers and their effect on calcium oxalate crystallization. BJU Int. 83: 919-923.

47. McCulloch RK, Bowyer RC, Brockis JW. (1981) Possible roles of urate in calcium urolithiasis. In: Brockis JG and Finlayson B (eds.) Urinary Calculus. PSG Publishing Company Inc., Littleton, Massachusetts; 347-352.

48. Grover PK, Ryall RL, Marshall VR. (1990) Effect of urate on calcium oxalate crystallization in human urine: evidence for a promotory role of hyperuricosuria in urolithiasis. Clin. Sci. 79: 9-15.

49. Grover PK, Ryall RL, Marshall VR. (1992) Calcium oxalate crystallization in urine: role of urate and glycosaminoglycans. Kidney Int. 41: 149-154.

50. Robertson WG, Knowles F, Peacock M. (1976) Urinary mucopolysaccharide inhibitors of calcium oxalate crystallization. In: Fleisch H, Robertson WG, Smith LH and Vahlensieck W (eds.) Urolithiasis Research. Plenum Press, New York and London; 331-334.

51. Grover PK, Ryall RL, Marshall VR. (1993) Dissolved urate promotes calcium oxalate crystallization: epitaxy is not the cause. Clin. Sci. 85: 303-307.

52. Kallistratos G, Timmermann A, Fenner O. (1970) Zum Einfluss des Aussalzeffektes auf die Bildung von calciumoxalatKristallen im menschlichen Harn. Naturwissenschaften 57: 198.

53. Kallistratos G, Timmermann A. (1971) The "salting-out" effect as a possible causative factor for the formation of calcium oxalate crystals in human urine. Paper presented at the 66th annual meeting of the American Urological Association in Chicago, Illinois, USA, May 17, 1971, Urological Research Forum. 